\title{
Dix ans de recherche par les résidents en anesthésiologie à l'Université de Montréal : un bilan
}

\author{
Michel GIRARD, Pierre DROLET
}

\begin{abstract}
Résumé Contexte: En 1989, la décision que tous les résidents en anesthésiologie de l'Université de Montréal devraient faire de la recherche fut prise. Des mesures pour encadrer cette activité dans un environnement porteur furent mises en place. Buts : Le but premier de ce travail est de dresser un bilan des activités de recherche auxquelles ont participé ces résidents depuis 1990. Méthode: Afin d'évaluer l'impact de tels stages, certains indices témoignant de la vitalité académique du département (publications, présentations, distinctions, pour les décennies allant de 1980 à 1989 et de 1990 à 1999) sont répertoriés et comparés, lorsque possible, aux résultats obtenus lors de la décennie précédente. Résultats : De 1980 à 1989, 85 résidents auraient été susceptibles de faire un stage de recherche sil avait existé. De 1990 à 1999, 51 résidents ont fait ce stage. Seuls les articles publiés dans des journaux avec comité de révision par des pairs peuvent être comparés. Le ratio "nombre de publications/nombre de résidents " est plus bas pour la période $80-89(0,13$, intervalle de confiance $95 \%: 0,07-$ 0,22) comparé à l'époque 90-99 (0,45, intervalle de confiance $95 \%: 0,31-0,60)$. Le bilan des autres activités académiques, qui n’ont pu être comparées, est présenté. Conclusion : La littérature indique que les programmes qui connaissent du succès dans ce type de projet ont certaines caractéristiques en commun. Les mesures mises en place lors de l'initiation du projet étaient conformes à ce que suggère la littérature.
\end{abstract}

Mots clés Résidents - recherche; activité académique ; programme de résidence.

Summary Background: In 1989, it was decided that all residents in anesthesiology at the Universite de Montréal should do research. Mesures were taken to create a favourable environnement for this project. Objective: The main goal of this work is to evaluate the output of the residents' research since 1990. Material: All documents available at the anaesthesiology university department, as well as results of a Medline search, for periods from 1980 to 1989 and from 1990 to 1999, were analysed. Results: If a research rotation had it been available from 1980 to 1989, 85 residents would have made one. From 1990 to 1999, 51 residents made a research rotation. For these two periods only articles published in peer reviewed journals can be compared. The ratio "number of publications/number of residents" is lower for the 80-89 period (0.13, confidence interval $95 \%: 0.07-0.22)$ in comparison to the 90-99 period (0.45, confidence interval $95 \%: 0.31-0.60$ ). The evaluation of other academic activities that could not be compared is also presented. Conclusion: Current evidence shows that residency programs which have success with this type of project share a number of characteristics. The actions put in place at the initiation of the project were in accordance with data found in the literature.

Key Words Residents ; post-graduate programs ; research ; academic activities.

Pedagogie Medicale 2001; 2 : 31-36.

Université de Montréal - Correspondance : Michel Girard - Hôpital Maisonneuve-Rosemont - Département d'anesthésie réanimation - 5415 boul. de l'Assomption - Montréal, QC. - Canada H1T 2M4 - Tél. : (514) 252-3426, télécopieur : (514) 252-3542 - courriel : michel.girard.2@umontreal.ca 


\section{Concepts et Innovations}

\section{Introduction}

De nombreux auteurs soulignent les avantages, pour un médecin en formation spécialisée, de participer à des activités structurées de recherche. De même, les résultats d'enquêtes menées auprès de directeurs de programme $^{1}$ et des résidents ${ }^{2}$ confirment les aspects positifs d'une telle participation. Cependant, plusieurs programmes hésitent à inscrire formellement des activités de recherche à leur curriculum et ce, même si ce type d'activité est souhaité par les grands organismes nord-américains d'agrément. Lors d'une visite d'agrément menée en 1985 par le Collège Royal des Médecins et Chirurgiens du Canada, les remarques suivantes étaient émises à l'endroit du programme d'anesthésiologie de l'Université de Montréal : " Il existe dans environ la moitié des milieux hospitaliers... des activités de recherche clinique auxquelles peuvent s'associer les résidents du programme. Toutefois, peu de résidents sont associés à ces activités soit par manque d'encouragement, soit par manque d'intérêt ". Des commentaires analogues étaient émis en 1988 lors d'une visite subséquente faite par le même organisme. Afin de remédier à cette situation, le comité pédagogique du programme d'anesthésiologie décidait, en 1989, de créer un stage de recherche formel d'une durée de 3 mois destiné aux résidents effectuant leur troisième année de formation (programme de 5 ans). Le but premier de ce travail est de dresser un bilan des activités de recherche auxquelles ont participé les résidents en anesthésiologie de l'Université de Montréal dans le cadre des stages de recherche menés depuis 1990. Afin d'évaluer l'impact de tels stages, certains indices témoignant de la vitalité académique du département (publications, présentations, distinctions) sont répertoriés et comparés, lorsque possible, aux résultats obtenus lors de la décennie précédente.

\section{Méthode}

Afin de recueillir les données nécessaires au bilan et à la comparaison, les sources suivantes furent consultées : rapports annuels d'activité du département universitaire d'anesthésiologie, curriculum vitae des professeurs et résidents (section portant sur les publications), le journal du département (Sarbacane), la base de données bibliographique Medline, les rapports du comité de recherche du département universitaire, une liste fournie par le président du comité de la recherche et la feuille sommaire des dossiers académiques des rési- dents pour la confirmation de la date de leur stage. Les données suivantes ont été recueillies : le nombre de résidents pour les décennies allant de 1980 à 1989 et 1990 à 1999 , le nombre de présentations formelles dans le cadre de forums provinciaux, nationaux et internationaux, l'attribution de prix associée à ces présentations, le nombre de publications (sous forme d'abrégé ou d'article), parus dans des journaux dotés de comités de révision par des pairs, faisant suite au stage ou portant sur un sujet autre que celui étudié lors du stage de recherche. Enfin, le nombre de descriptions de cas cliniques et de lettres à l'éditeur est noté. Afin d'établir une comparaison entre la décennie précédant l'introduction des stages de recherche (80-89) et la période suivant leur inscription au curriculum (90-99), le ratio nombre de publications/nombre de résidents est calculé et comparé pour chaque époque.

\section{Résultats}

Le nombre de résidents présents dans le programme au cours de la décennie 1990-1999 est inférieur au nombre de résidents de niveau académique équivalent au cours de la décennie précédente (tableau 1). Il n'y a pas de données disponibles quant au nombre de présentations ou d'abrégés au cours de la décennie allant de 1980 à 1989. Le nombre de présentations et de distinctions obtenues au niveau provincial, national et international au cours des années 1990 à 1999 se trouve au tableau 2. Le nombre total d'articles publiés dans des revues avec comité de révision par des pairs pour les périodes 1980 à 1989 et 1990 à 1999 est rapporté au tableau 3. Le ratio "nombre de publications/nombre de résidents " est plus bas pour la période 80-89 (0,13, intervalle de confiance $95 \%$ : 0,07-0,22) comparé à l'époque 90-99 (0,45, intervalle de confiance $95 \%: 0,31-0,60)$. Le nombre d'abrégés, articles (faisant suite ou non-associés au stage de recherche), rapports de cas cliniques et lettres à l'éditeur, publiés durant la période 1990-1999 (tableau 4). Les données pour les années 1998 et 1999 sont incomplètes, plusieurs travaux n'étant pas complétés à ce jour.

\section{Discussion}

La participation des résidents à des activités de recherche peut se justifier par des exigences réglementaires, ce qui permet de répondre aux normes d'agrément des organismes accréditeurs nord-américains. 
Tableau 1 : Nombre de résidents de niveau équivalent au cours des décennies allant de 1980 à 1989 et de 1990 à 1999

\begin{tabular}{|c|c|c|c|c|c|c|c|c|c|c|}
\hline & $80 / 90$ & $81 / 91$ & $82 / 92$ & $83 / 93$ & $84 / 94$ & $85 / 95$ & $86 / 96$ & $87 / 97$ & $88 / 98$ & $89 / 99$ \\
\hline \multicolumn{11}{|l|}{1980} \\
\hline à & 7 & 4 & 11 & 8 & 9 & 11 & 10 & 10 & 8 & 7 \\
\hline \multicolumn{11}{|l|}{1989} \\
\hline \multicolumn{11}{|l|}{1990} \\
\hline à & 3 & 1 & 5 & 6 & 2 & 6 & 3 & 9 & 8 & 8 \\
\hline 1999 & & & & & & & & & & \\
\hline
\end{tabular}

Tableau 2 : Nombre de présentations et de distinction obtenues au niveau provincial, national et international au cours des années 1990 à 1999

\begin{tabular}{|c|c|c|c|c|c|c|c|c|c|c|}
\hline Année & 90 & 91 & 92 & 93 & 94 & 95 & 96 & 97 & 98 & 99 \\
\hline Provincial & 1 & & 4 & 4 & 2 & 3 & 2 & 4 & 2 & \\
\hline Distinction & & & & 2 & 1 & & 1 & 1 & 2 & \\
\hline National & 1 & & 1 & 1 & 1 & 1 & & 4 & 3 & \\
\hline Distinction & & & & 1 & 1 & & & 1 & & \\
\hline International & & & & 2 & & 3 & 1 & 9 & 7 & 1 \\
\hline Distinction & & & & & & & 1 & & & \\
\hline Total : & 2 & 0 & 5 & 7 & 3 & 7 & 3 & 17 & 12 & \\
\hline
\end{tabular}

\begin{tabular}{|c|c|c|c|c|c|c|c|c|c|c|}
\hline \multicolumn{11}{|c|}{$\begin{array}{c}\text { Tableau } 3 \text { : Nombre d'articles publiés par des journaux avec comité de révision } \\
\text { par les pairs pour les périodes } 1980 \text { à } 1989 \text { et } 1990 \text { à } 1999\end{array}$} \\
\hline & $80 / 90$ & $81 / 91$ & $82 / 92$ & $83 / 93$ & $84 / 94$ & $85 / 95$ & $86 / 96$ & $87 / 97$ & $88 / 98$ & $89 / 99$ \\
\hline 1980 & & & & & & & & & & \\
\hline $\begin{array}{c}\text { à } \\
1989\end{array}$ & 0 & 1 & 1 & 3 & 0 & 0 & 3 & 2 & 0 & 1 \\
\hline 1990 & & & & & & & & & & \\
\hline $\begin{array}{c}\text { à } \\
1999\end{array}$ & 0 & 1 & 2 & 5 & 2 & 2 & 2 & 7 & 2 & \\
\hline
\end{tabular}




\section{Concepts et Innovations}

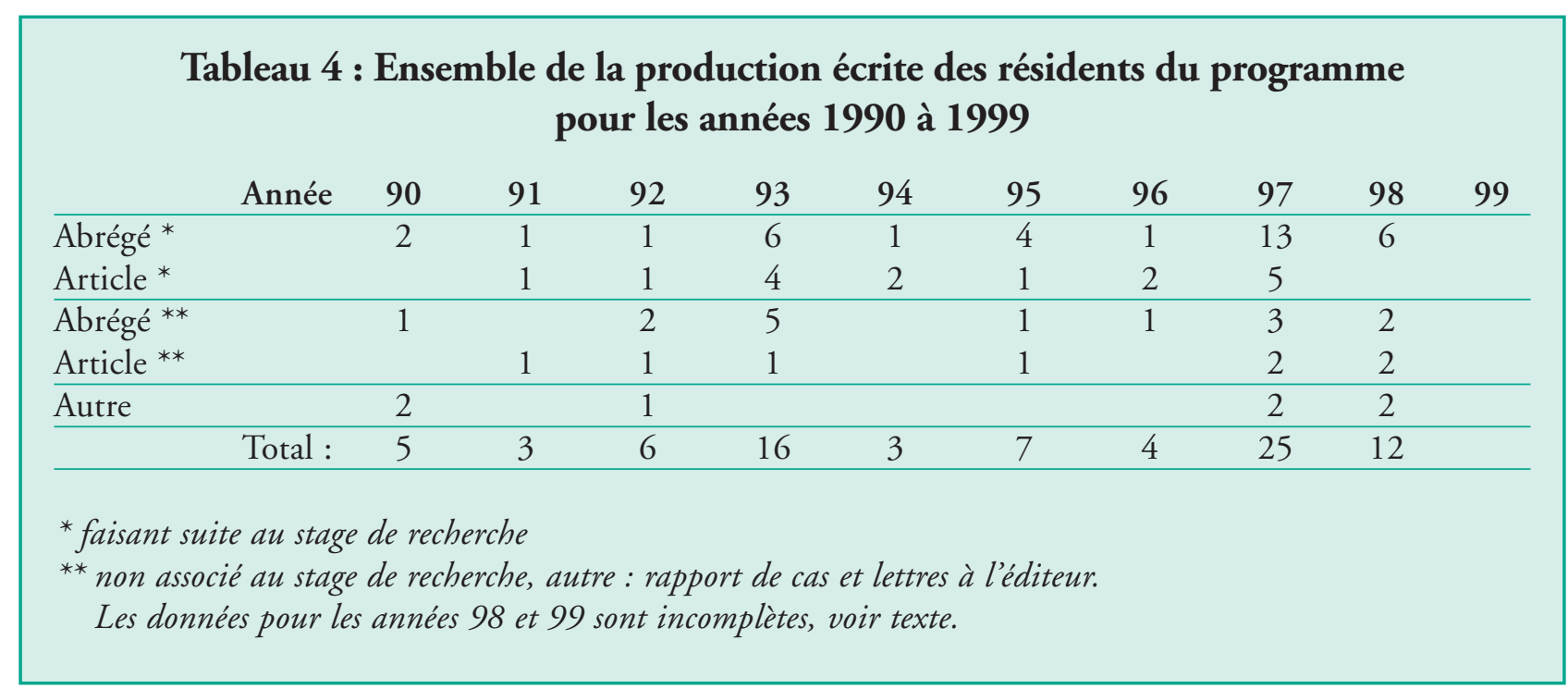

Tableau 5 : Balises initiales imposées par le comité pédagogique du département universitaire d'anesthésiologie de l'université de Montréal quant aux principales caractéristiques des projets de recherche

\footnotetext{
- Projets proposés et supervisés par les professeurs

- Document écrit, un an à l'avance

- Projets de recherche clinique ou fondamentale sont acceptés

- Projet pouvant être complété en trois mois

- Pas de projet déjà en cours

- Pas d'études rétrospectives

- Pas de gardes (astreintes) durant le stage de recherche
}

Toutefois, au-delà de ces exigences, certains auteurs décrivent des raisons d'un tout autre ordre pour appuyer cette pratique. Gurd ${ }^{3}$ croit que la participation à des activités de nature académique est une des caractéristiques qui différencie les écoles de médecine des écoles de métier. Pour Lentle $e^{4}$, il s'agit d'une occasion unique d'acquérir et/ou développer la curiosité et les techniques nécessaires au développement d'habitudes menant à l'apprentissage à long terme. Chung ${ }^{5}$ croit que l'esprit critique qu'aide à développer ce type de formation aide à limiter l'empirisme en pratique clinique. De plus, il a été démontré, en radiologie ${ }^{6}$, que la participation à des activités de recherche durant la formation augmente le taux de rétention des résidents en milieu académique une fois la formation terminée. Enfin ce retour en milieu académique s'accompagne d'un taux plus élevé de participation des nouveaux cliniciens à des activités de recherche $e^{7}$ s'ils ont participé à des activités de recherche au cours de leur formation. Enfin, qu'il nous soit permis de spéculer sur l'impact qu'ont ces projets sur l'attitude des résidents durant leur formation. En effet, le tableau 4 nous indique que bon nombre de présentations et de publications ont été faites sans qu'ils soient issus du travail fait durant les stages de recherche. Nous croyons que ce phénomène est indicateur d'un accroissement de l'intérêt des résidents pour la recherche mais cette hypothèse reste à démontrer.

La participation obligatoire à des activités académiques, qu'il s'agisse d'activités de recherche ou de travaux d'érudition, varie selon les programmes et les différentes spécialités médicales, les statistiques disponibles étant essentiellement américaines. Les rési- 
dents sont tenus de pratiquer de telles activités dans $64 \%$ des programmes de gynécologie-obstétrique ${ }^{8}$, $68 \%$ des programmes de sous-spécialités ${ }^{1} 15 \%$ des programmes de médecine familiale ${ }^{1}, 19 \%$ des programmes de radiologie et $28 \%$ des programmes de pédiatrie ${ }^{10}$. L'organisme accréditeur américain, en médecine interne, exige que ce soit le cas dans tous les programmes sous son autorité ${ }^{11}$. Bien que de nombreux programmes de formation exigent que leurs résidents participent à des activités de nature académique, la nature des activités acceptées comme répondant à cette exigence varie. Ainsi pour certains, un rapport de cas clinique suffit, alors que pour d'autres plus d'un projet devra être fait au cours de la formation, un de ceux-ci devant être de nature prospective ${ }^{8}$. La publication des résultats est exigée par certains programmes. Nous avons choisi de ne pas l'exiger, mais plutôt de mettre l'emphase sur un environnement académique aussi enrichissant que possible. Il nous parait y avoir trop d'embûches, hors du contrôle d'un résident effectuant un stage de 3 mois, pour exiger la publication d'un article. Toutefois, de plus en plus d'emphase est placée sur la présentation des résultats, à tout le moins, au niveau provincial.

Pour qu'un stage de recherche atteigne ses objectifs et soit la source d'une activité académique soutenue et profitable pour les résidents, il ne suffit pas qu'il figure sur la liste des stages obligatoires. Ainsi, en plus de créer un stage de recherche pour ses résidents, le comité pédagogique a pris la décision d'encadrer ces stages par un ensemble de conditions (tableau 5). À ces conditions initiales sont venues s'ajouter au cours des années des activités gravitant autour de ces projets. Un cours d'épidémiologie a été organisé dès 1992. Il s’agit d'un cours de deux jours donné durant la première ou deuxième année de formation. De plus, afin de fournir une tribune où présenter et défendre leurs travaux, une " Journée des résidents » est organisé annuellement avec une autre université depuis 1992. Des prix sont accordés aux meilleures présentations. Nos résidents sont également encouragés à soumettre leurs travaux lors de congrès nationaux ou internationaux. Les différents hôpitaux et le département universitaire partagent les frais encourus pour les présentations hors de Montréal, lorsqu'il s'agit de congrès d'associations savantes reconnues. En 1995, les objectifs du stage ont été publiés. Un quatrième mois de stage optionnel en recherche fut ajouté en 1997. Depuis 1999, les propositions des maîtres de stage sont consignées dans un registre regroupant un ensemble d'informations pertinentes à ce stage et tous les résidents sont reçus par les membres du comité de la recherche de façon à suivre l'évolution de leur projet.

Peu d'auteurs ont étudié les facteurs qui favorisent le succès de telles entreprises. Schultz ${ }^{12}$, dans un éditorial accompagnant un travail ${ }^{11}$ étudiant ces facteurs et portant sur des programmes de médecine interne américains, résumait les principales caractéristiques des programmes où on a pris les moyens pour favoriser l'association des résidents à des activités académiques (tableau 6). Dans un travail évaluant les programmes de médecine familiale américains DeHaven ${ }^{13}$ arrive aux mêmes conclusions, tout en ajoutant qu'il est préférable de commencer tôt durant la formation et que les projets de recherche clinique sont mieux acceptés que les travaux fondamentaux car l'étudiant y voit un lien

\section{Tableau 6 : Principales caractéristiques des programmes de médecine interne américains où l'organisation de stages de recherche est un succès ${ }^{12}$}

- Un directeur de la recherche qui organise et supervise professeurs et résidents

- Des objectifs et un échéancier clair

- Des projets précis et limités dans le temps

- Curriculum départemental en méthodologie, sciences de l'information et en communication

- Temps dédié au projet

- Tribune pour que le résident puisse présenter

- Le plus important : des professeurs enthousiastes 


\section{Concepts et Innovations}

avec la pratique. Pour nous, la présence d'un médecin en mesure de procéder aux analyses statistiques lors des projets est un autre facteur qui contribue au succès de ces projets.

Nos résultats montrent qu'il est possible de rehausser de façon importante la participation des médecins en formation à des activités de recherche et de publication. Les conditions établies au lancement de notre projet (tableau 5) sont comparables à celles décrites par
Schultz (tableau 6) et DeHaven et nous paraissent fournir un cadre conceptuel raisonnable pour promouvoir la recherche chez les résidents.

\section{Remerciements}

Les auteurs remercient Mme Johanne Godin pour son aide précieuse lors de la saisie des données.

\section{Références}

1.DeHaven MJ, Wilson GR, Murphee DD, Grundig $J P$. Family practice residency program directors' views on research. Fam Med 1997; 29 : 33-7.

2.Stewart RD, Doyle J, Lolis SS, Stone MD. Surgical resident research in New England. Arch Surg 2000 ; $135: 439-44$

3. Gurd FN. Scholarship and science and the Royal College. Ann R Coll Phys Surg Can 1986; 19 : 461-4.

4.Lentle BC. The place of research in medical education and practice. Ann R Coll Phys Surg Can 1986; $19: 423-4$.

5.Chung R, Diaz J, Li P. A method of teaching clinical research in a community hospital residency program. Am J Surg 1999; 177 : 83-85.

6. Hillman BJ, Nash KD, Witzke DB, Fajardo LL, Davis D. The RSNA-AUR-ARRS introduction to research program for 2 nd year radiology residents : effect on career choice and early academic performance. Radiological Society of North America. Association of University Radiologists. American Roentgen Ray Society. Radiology 1998 ; 209 : 32326.

8.Sulak PJ, Croop JA, Hillis A, Kuehl TJ. Resident research in obstetrics and gynecology : development of a program with comparison to a national survey of residency programs. Am J Obstet Gynecol 1992 ; 167: 498-502.

9. Hillman BJ, Fajardo LL, Witzke DB, Cardenas D, Irion $M$, Fulginiti JV. Influences affecting radiologists' choices of academic or private practice careers. Radiology 1990 ; 174 (2): 561-4.

10.Brouhard BH, Doyle W, Aceves J, McHugh $M J$. Research in pediatric residency programs. Pediatrics 1996; 97: 71-3.

11.Alguire PC, Anderson WA, Albrecht RR, Poland GA. Resident research in internal medicine training programs. Ann Intern Med 1996 ; 124 : 321-8.

12.Schultz HJ. Research during internal medicine residency training : meeting the challenge of the residency review committee. Ann Intern Med $1996 ; 124: 340-2$.

13.DeHaven MJ, Wilson GR, O'Connor-Kettlestrings $P$. Creating a research culture: what we can learn from residencies that are successful in research. Fam Med 1998 ; 30 : 501-7.

7.Ledley FD, Lovejoy FH, Jr. Factors influencing the interests, career paths, and research activities of recent graduates from an academic, pediatric residency program. Pediatrics 1993 ; $92: 436-41$. 